Masuma H. Mammadova

DOI: $10.25045 /$ jpit.v07.i2.02

Institute of Information Technology of ANAS, Baku, Azerbaijan

masuma.huseyn@iit.ab.az

\title{
BIG DATA IN ELECTRONIC MEDICINE: OPPORTUNITIES, CHALLENGES AND PERSPECTIVES
}

The paper reviews the factors defining excessive growth of the information in medicine. The specific peculiarities of medical data are investigated; the essence of Big Data phenomenon and its potential in electronic medicine are defined. Big Data applications are systematized, and their capacity for the support of medical and diagnostic, and management decisions is shown. Advantages of Big Data-analytics in this sphere are indicated in the context of development and transformation of medicine. The challenges, limiting the usage of Big Data in medicine, and the standpoint directions of its application in the given subject field are specified.

Keywords: Big Data, e-medicine, health data, Big Data-applications, decision-making, Big Datachallenges.

\section{Introduction}

With the emergence and rapid development of information and communication and network technology, the world is confronted with an avalanche increase of the information received in a society called the "information explosion" [1]. The volume of generated information is growing exponentially, and today, approximately $90 \%$ of the data in the world has been created in the last two years [2]. In accordance with a new study Digital Universe, conducted by the research firm International Data Corporation (IDC) [3], by the next 8 years, the volume of data throughout the world is predicted to reach 40 zettabytes. This means that 5,200 gigabytes of data will be accounted for each person on the Earth. At the same time, IDC estimates that today less than $1 \%$ out of all available information has been analyzed, and at least, $20 \%$ has been protected.

Unprecedented growth of information, only a tiny part of which is used worldwide, demonstrates an understanding of the indisputable fact that the effective use of the increasing amount of information should be one of the greatest scientific and technical challenges of the XXI century [4].

Medicine is one of the industries that historically has been generating a large amount of data, traditionally managed by accounting, compliance with regulatory requirements and criteria for the quality of services delivered to the patients [5]. At the same time, medicine belongs to the category of spheres of activity, which most lagging on a number of client-oriented industries (banking, retail, etc.) for the use of IT and, accordingly, Big Data. For the first time, medicine information technology was applied in the 60's of the previous century to collect and process large amounts of different statistics. However, the amount of medical data has been significantly increased only during the last 15-20 years, due to the transition to digital format. Despite storing a large part of the medical data in paper form, the tendency of rapid digitization has facilitated the accumulation of terabytes of data [4]. According to some estimations, the volume of medical data in 2012 reached about 500 petabytes [6]. Recent studies show that more than $30 \%$ of all data stored in the world is represented by medical information, and in the future, this share is expected to rapidly increase [7]. According to forecasts, by 2020, the amount of medical data will reach 25,000 petabytes [8].

Huge streams of medical data provide great opportunities for the development of methods and applications for expanded analysis of the latter. Indeed, the real value of this stream can only be understood in the case of promoting the information extracted from the data to improve the quality of medical services $[9,10]$.

This paper aims at studying the potential of Big Data phenomenon in medicine as the means of improving the quality of the healthcare services through the possibilities of analytical support of medical and diagnostic, organizational and administrative decisions. 


\section{Key elements that define the information explosion in medicine}

According to [11] medical data is mainly generated due to the following three key elements: a set of personal Electronic Health Records (EHR), biotechnology, which laid the foundation of personalized medicine, and research and development ( $R$ \& D). This list is supplemented by two more indisputable key elements that ensure the generation and rapid growth of information, which includes advanced information and communication technologies (ICT) and the Internet.

$\boldsymbol{E H R}$ (electronic health record) [12] integrates electronic personal health records related to one person, collected and used by several health organizations. In the Russian literature, an analogue of EHR is integrated electronic card of patient (IECP) [13]. In fact, IECP consolidates personal medical records of the patient accumulated in electronic health cards (EHC) of separate healthcare organizations. The term EHC is an analogue of the international term Electronic Medical Record (EMR), and it is an electronic version of the patient's history.

Biotechnology, which caused a boom in molecular medicine, has stimulated the rapid growth of the volume of data, in particular personal genome, in biology and medicine. Further development of the science and technology has led to exponential decrease in the cost of genome-wide analysis, and has contributed to the explosive growth of data specific to a particular individual. Therefore, in 2012. The cost of decoding the human genome sequence fell below 1000 dollars, whereas in 2003, this figure was about 40 million dollars. As a result, this procedure became available to the masses [14]. Since the failure of gene structure is one of the causes of any disease, the gene therapy can enhance the effectiveness of the prescribed treatment by reducing the negative effects on the body. With the help of Big Data technologies, human genetic code can be compared to other medical conditions, and it is possible to exactly find out how their genes correlate with diseases. The study of the genetic characteristics of an individual is one of the fundamental elements of personalized medicine, the main purpose of which is the providing a certain drug to a particular patient in appropriate doses and at a particular time $[15,16]$.

Big Data Sources also include other biometric data, such as scanned fingerprints, handwriting, retina, X-ray and other medical images, and the values of vital signs (blood pressure, pulse, etc.).

$(\boldsymbol{R} \boldsymbol{\&} \boldsymbol{D})$. Medicine refers to one of those areas where a rapid development of science and technology is observed, and the results of scientific discoveries and developments are manifested quite clearly. The world is now facing a new challenge as an exponential growth rate of medical knowledge discoveries. Today, available biomedical literature catalogs include more than 18 million articles, with more than 800 thousand of them were cataloged in 2008. The rate of replenishment of medical literature doubles every 20 years, and since 2012, the number of annual revenues has exceeded 1 million [17].

An analysis of the dynamics of past 150 years, during which the effects of industrialization and information explosion manifested most acutely, shows that a typical doctor of general aid should be aware of about 10 thousand of diseases and syndromes, 4 thousand drugs, among which there are more than 2 thousand interactions, which define the possibility of sharing, and 1,1 thousands of laboratory tests [18].

Processing technology of large data can assist in the systematic analysis of unlimited volumes of multi-type medical information.

ICT and the Internet. Uninterruptedly growing rate of introduction of ICT in medicine does not only give a powerful impetus for change in this area, but also becomes the key tools in its transformation. This is accompanied by the rapid development of ICT itself. On the one hand, followed by the emergence of new platforms, hardware and software, network technology, models of data acquisition, storage, processing and analysis, on the one hand, emerging new sources of information generation.

Certainly, the Internet is an important source of health information, providing people with previously unavailable possibility of acquiring knowledge about diseases and medicines, diagnosis clarifying, the search as an effective treatment and as a physician of certain specialty, etc. According to the survey carried out in 2010 by the international consulting and marketing agency Manhattan Research LLC, the half of the patient-respondents surveyed the use of the Internet to search for medical information on health, diseases and their prevention, medicines, and etc. [19]. At the same time, 9 out of 10 patients make decision on their own treatment, based on data from the network. At once, the 
Internet plays a role of basic infrastructure in the generating processes, and in a reliable and secure transmission of medical information.

According to Cisco, in 2012, third of 2.8 zettabyte of transmitted information was accounted for the data automatically generated by the equipment and devices connected to the Internet or to the Internet of things. Cisco IBSG forecasts that, by 202050 billion devices will be connected to the Internet, despite the fact that these prognoses do not take into account the rapid development of the Internet technology and devices [20]. Besides the development of the Internet of Things and its further evolution, namely the Internet of Everything, which enables machine-to-machine (M2M), there is another powerful source of data generation [21]. This is an availability of networked portable devices (remote sensors and sensors of physical activity, diagnostic tools of public use, and etc.) [22], monitoring the status of patients' health. Adjustable electronic sensors produce millions of transactions per second, and therefore, reliable solution is required that can convert, save and work in real time. Certainly, it is possible with big data technologies.

The rapid development of mobile wireless communication and applications has stimulated the development and distribution of the methods of their innovative application to address the priorities of medicine and healthcare. This has led to the formation of a new field of e-health, known as Mobile Health (mHealth) [23]. According to the International Telecommunication Union (ITU), today, the number of wireless subscribers is reaching 5 billion people in the world, and over $85 \%$ of the world's population is in the coverage of commercial wireless network signal [24]. A large part of the data produced in the period from 2012 to 2020 , will not be generated by people, but by various types of devices in the course of their interaction with one another and with data network (sensors, smart phones, radio frequency identification devices (RFID), satellite navigation systems etc.) [25].

\section{Definition and essence of the concept of "Big Data" in medicine}

Before turning to the study of the concept of Big Data in the context of e-health, the definition of the term should be explained?

Analysis of the literature shows that, today, the term Big Data has no strict and universally accepted definition. According to [26], Big Data is the set of so voluminous and complex data sets that its handling with traditional tools in a reasonable time is quite difficult. McKinsey Institute [4] reports that the term Big Data means a set of data a size of which is beyond the capabilities of typical database for the collection, storage, management and analysis. Currently large-scaled data is considered a volume of which reaches 1 terabyte $(1$ terabyte $=1024$ gigabytes $)$ or more. Big Data is usually measured with the terms as "terabytes, petabytes, exabytes, etc." (1 exabyte $=1$ billion gigabytes). The report submitted in August 2012 in the US Congress, proposed the following definition: Big Data is the large volumes of data of high velocity, complex, and variable data that require advanced techniques and technologies to enable the capture, storage, distribution, management and analysis of the information" [10]. Another definition of the term is given in [27]: "Big Data -is a collection of tools and methods for processing the structured and unstructured data, huge amounts of different sources subjected to constant updates, in order to improve the quality of decision-making management, and to create new products and improve the competitiveness."

Stating from the above-mentioned definitions, the essence of Big Data is to enable high-velocity analysis of large volume of diverse data rapidly updated through new methods and technologies of data processing in real time. Big Data processing aims to identify the relationship between all the unrelated data and the identification of new hidden knowledge needed to support decision-making, to create new products, to improve the competitiveness of enterprises, etc.

Let's revise the definition, specificity and the nature of Big Data concept in the context of ehealth. To do this, first of all, the term "e-health" and "e-medicine" should be explained, the interpretation of which has multiple approaches [28, 29], and the author's understanding on these concepts should be clarified.

Accordingly, by definition, healthcare is a branch of state authority aimed to organize and provide affordable health care for the population, and to preserve and improve health rate [30]. In other 
words, the basic functions of the industry are the institutional (administrative) and managerial functions oriented at data analysis, forecasting and effective planning of the future development based on representative statistical samples, time series, and so on.

Medicine is a system of scientific knowledge and practices, united in order to define, treat and prevent disease, and to preserve and promote the health and ability of people prolong their lives [30]. Medicine, as a science of healing, encompasses a gigantic system of ever-increasing knowledge and data, the volume of which makes it difficult to work with them. Therefore, the use of modern technologies for processing the medical knowledge and data, which provide the opportunity to support therapeutic and diagnostic decisions, is vital for medical institutions. The main priority of the digitization should be the task of medical support activities that directly determines the quality of medical services, but not taking into account the problem of statistical indicators [31]. Based on the objectives of medicine and agreeing with those authors, who believe that informatization should aim to support medical and diagnostic solutions, and information is important for doctors first of all for the treatment of the patient, rather than including it into the accounting system, this paper prefers the term "e-health". Therefore, this paper discusses the phenomenon of Big Data in electronic (digital) medicine, considering this term in a broader sense, including organizational and managerial processes of the healthcare of the population.

By definition, Big Data in medicine - is rapidly rechargeable various electronic medical data of a colossal volume that cannot be managed by traditional tools and methods, software and/or hardware [32].

\section{Key indicators of Big Data in medicine}

The most common disclosure of the phenomenon of Big Data is by specifying the problems encountered at the present stage of technological development in data processing [33, 34]. The main problems are pertained to Big Data include Volume, Variety and Velocity. For some areas of activity, including medicine, researchers and practitioners introduced two more characteristics: Veracity and Value of Big Data [35]. The close relationship of these problems should be noted, and their interpretation in medicine should be given.

Volume of medical data. Continuous generation and accumulation of medical information in the coming years will lead to an incredible amount of data [10]. Currently, medical data includes clinical data provided by physicians, as well as personal health records of patients (EHR), radiology images (X-ray and mammography images), MRI (magnetic resonance imaging), CT (computer tomography), and etc., and laboratory and pharmacy data, insurance claims and so forth. At the same time, a huge amount of medical information that is not specifically related to the patient is also generated. These are numerous medical publications, research reports, the results of research and development (R\&D) activities, surveys, etc. New types of medical data such as multidimensional 3D/4D imaging, genomics, biometric sensor records, etc., also contribute to the exponential increase of data. The volume of this data is very large, the results of one study can occupy hundreds of megabytes. Even annual refillable data of one clinic associated with advanced diagnostic methods can reach terabytes or even tens of terabytes. For example, in the US the volume of medical data was 150 Exabytes in 2011 [36].The complexity and often inability of both processing and storing Big Data, as well as establishing meaningful links and arrangements between them to extract useful information requires the use of new technology and algorithmic solutions.

Variety. Simultaneous processing of medical data of various formats (structured, semi-structured and unstructured) is one of the specific problems of medicine [36]. More than 70-80\% of medical data are unstructured. Furthermore, there is a steady upward trend in the information rate, relatively, to that of structured data. For diagnostic and treatment decisions, there is a need to integrate clinical information and biological data that are in different formats and generated from different heterogeneous sources. For example, medical information may be presented in the form of numerical values in arbitrary units, images, texts, handwritten notes and doctor's prescriptions, gene and protein sequences and so forth. Joint storage, comparison and conversion of different data types require solving very complex problems such as image recognition, data compression, etc. Here, medicine does not always take advantage of ready- 
made solutions from other areas [37]. Technology for comprehensive analysis of medical data of various formats is needed, which enables them to be useful for further processing.

Velocity of generated medical data. A constant stream of new data is accumulated at an unprecedented rate, i.e., observed growth of data volume and variety is directly related to the rate at which they are generated. Specificity of the problem in medicine is that the rate of data replenishment also limits Big Data processing in medicine. Thus, the information from the devices, tracking the patients in intensive care, enters continuously, i.e. in real time, and it requires immediate processing and analysis for the timely development of preliminary diagnosis $[10,36]$.

Veracity. This feature reflects the semantic and syntactic definition, quality, relevance and reliability of the data. A number of issues to ensure the reliability of data are specific to medicine, as they deal with the diagnosis, treatment methods, recipes, procedures, etc. Therefore, Big Data analysis must be error-free and reliable, since a person's life may depend on the trustworthiness made by Big Data analysis. On the other hand, the poor quality of health data, especially unstructured one, is one of the major problems: medical cards filled with errors, misinterpretation and inaccurate digital input of medical appointments of the doctor in recipes, due to poor handwriting, are classified as the most common examples. Veracity of data may also depend on the quality of medical devices and sensors (e.g., wires may break and the signal may be weak due to the wrong placement of the sensor; and electrical noise may also affect the performance) $[36,38]$.

Too high value of the failure causes distrust of the medical community and the results of Big Data analysis.

Value of stored data. This feature, representing the interest for different parties and decisionmakers, characterizes Big Data in terms of their usefulness and bringing a certain value to the medical facility (MF) and healthcare system as a whole (e.g., improvement of business processes, defining business strategies, cost optimization, etc.) [35].

With the rapid growth of data volume the models for healthcare and treatment costs will also vary. Although profit is not and should not be the main motivator, but it is vital for healthcare organizations to purchase available tools, infrastructure and the methods for effective use of Big Data. Otherwise, MI (clinics, hospitals, home health care services, rehabilitation centers, etc.) are potentially at risk of losing millions of dollars in revenue and profits [37, 39].

The analysis of characteristics of medical information on the one hand, and specific features Big Data on the other, allows to conclude about the appropriateness of the latter to handle vast amounts of medical data. Thus, the potential and the essence of Big Data in medicine, in our view, can be defined as: 1) providing storing, sharing, rapid processing and analysis of the continuously generated heterogeneous and multi-format data coming from different sources by the latest technologies and tools; 2) identifying the correlations between different and seemingly unrelated medical conditions and the factors affecting them; 3 ) obtaining the results of processing and analysis in the form of information (knowledge), understandable to the doctor and ready to make substantiated decisions.

\section{Application areas and potential of Big Data in medicine}

During the last decades, along with an upward tendency of health care efficiency, the rocketing health care costs have been observed. The problem is actualized in connection with the continuing aging of the population in many developing and developed countries and with the rapid growth in the age cohort of citizens over the age of 65 , with the prevalence of such "lifestyle diseases" such as obesity and diabetes, and for the cohort of older - memory loss, arthritis and etc. However, almost exhausted possibilities of extensive development of the health care system by increasing its volume, and its costs respectively, have no prospects. In this regard, today, new conceptual approaches are developed to solve this problem, considering Big Data as an additional source of compensation for costs on the one hand, and to improve the quality of medical services - on the other. 


\section{The role of Big Data-analytics in medicine}

Analytics is a tool or a set of techniques that transform raw data into useful information. Big Data as an analytical basis for new IT applications in the medical industry can be used to support both medicaldiagnostic, and organizational and management decisions. For example, such information can be used by health organizations to support important strategic and operational decisions, to optimize expenses, to provide long-term forecasting, and to create additional value. With the development of technologies and tools of Big Data analytics, medical organizations can easily manage the enormous amount of diverse and multi-format digital data from various sources. Here, the analytics plays very important role in the separation of useful information from the worthless [40]. The patient-oriented approach and Big Data as an analytical basis for new IT solutions in medicine has a potential to transform almost every field of the industry. Analysis of the literature allows us to identify a number of areas of Big Data applications in medicine, promising from the standpoint of the potential of the latter.

\section{Big Data to support decision-making on management of healthcare quality}

An increasing availability of medical data allows offering a enhanced understanding of the relationships that underlie the vast amount of information from different data sets and transforming the latter into new knowledge with the use of Big Data technologies. As the result of the analysis of Big Data, it is possible to identify such unexpected relationships or patterns that are not detectable by a human. For example, Big Data technologies allows simultaneously handling the databases of health records of a patient, genomic data and medical research reports and ultimately giving useful information for the best solutions with respect to the treatment of a specific patient [38].

In essence, the terms "analysis of Big Data" and "decision-making" are the implementation of process of careful analysis of a huge amount of big data to identify certain trends and making vital decisions [41].

Big Data in medical decision support. McKinsey estimates that the introduction of Big Data analysis techniques in medicine can save the US health care for about 300 billion dollars a year. The savings will work due to more timely and accurate diagnosis, appointment of appropriate treatment, and reduction of expenditure on research. It is expected that treatment efficiency will be improved by processing of all available information. Doctors will use new generation decision support systems and expert systems that provide physicians with unprecedented access to the practices of colleagues through the EHR analysis of patients both in different geographical locations and in the country. This, in turn, will enable to minimize the subjective human factor in making medical decisions about the patient's treatment strategies.

To confirm the viability of this forecast it is enough to note that the expert system (ES) Watson IBM passed the exams on a common basis in 2013 and received a medical diploma, acquiring the legal right to treat people. ES is already showing exceptional results in the field of oncology. It is able to examine the patient's medical records, records and comments of the doctors, to view the latest research on this topic and to offer a diagnosis based on all the studied data. At the same time, IBM Watson analyzes the data in details, compares various factors and draws analogies. As the initial data more than 600 thousands of medical findings and diagnoses, 2 million pages of texts taken from 42 medical journals, and the results of clinical trials in oncology are loaded into a supercomputer memory. Watson can "analyze" 1.5 million medical records of different patients, and identify the most suitable treatment methods in each certain case based on the records data of successful fight against these diseases (best practices) [42].

Currently, one of the trends of acquiring significance is clinical decision support for the management of the individual patient's health based on the aggregate data of the population as a whole. Moreover, unique advantage of Big Data analysis technologies can be very useful here, which is expressed in their ability to analyze the data of the entire population based on various demographics, as well as to segment the population by individual cohorts. Therefore, today, the data of specific patients over the past year scan be compared to the results of ongoing laboratory research with the use 
of Big Data technologies within the same system, and additionally, this information can be linked to demographic data, retrospective information on drug reactions and drug interactions of the patients of any age, gender and ethnic background. These features of Big Data enable to build both the geographic and social model of health of the population, as well as the predictive development models of epidemic outbreaks, providing decision support for the prevention of the latter [43].

Big Data potential in the standardization of medical decisions. Each patient in his practice more than once has confronted with a situation where two different doctors very differently have interpreted the results of his tests or the same radiological image (medical data) and have made different diagnoses. Analysis of Big Data, i.e. accumulated statistical data on certain diseases, and its comparison with the current data of a particular patient (results of lab tests, X-rays, etc.), as well as with his EHR can be used for medical decision support. Minimizing the personal human factor in the process of comparison of the patient's heterogeneous data, his/her symptoms and the sheer volume of statistics on certain diseases provides the physician to select the best solution, which corresponds to the best diagnosis, i.e., promotes the standardization of medical decisions. In clinical practice, there is often a need for standardization of medical solutions. For example, in the analysis of medical errors, litigation, medical expertise, wrong diagnosis and treatment, identification of the effectiveness of prescribed medications in each case (disease). In these situations, Big Data can provide informed decisions support (revealing the truth) [44].

Big Data contribution to the creation and development of personalized medicine. Studies show that, in the near future, the quality of medical decisions will not and will not be capable to rely on an experience and intuition: the competitive advantages will be achieved by predicting the consequences of decisions, which actualizes the need to create a fundamentally new model of health care organization, so-called personalized medicine) based on the concept of 4P. The medical concept of 4P integrates the notions of personalization (individual approach to each patient taking into account their genetic characteristics), predictive (predicting the vulnerability to the developing disease), preventive (preventing or reducing the risk of the developing disease), participative (motivated participation of the patient in the prevention of possible diseases and their treatment) [45]. The essence of the personalized medicine is the individualization of drug therapy in accordance with the personal data and genotype of the particular patient. As a new paradigm in the healthcare, personalized medicine involves early (preclinical) detection of diseases at the stage of prediction of predisposition and subsequent preventive measures [45, 46].

Big Data technologies can play a significant role in the realization of personalized medicine, which is focused on choosing the right treatment. Hence, Big Data-based decision support systems can predict the response of the patients to certain drugs and assign absolutely unique drugs in individual doses grounding on processing and analysis of huge volumes of their genetic information [46].

The process of medicine personalization, i.e. the transition from physician-oriented approach to providing medical services to the patient-centered based on individualized medical care, is already taking place [47]. For example, more than $20 \%$ of new drugs, food and medicines approved in 2014 by the US Personalized Medicine Coalition (founded in 2004), was the production of personalized medicine [48]. In some US clinics, genotype database is integrated with the system of EHR drug prescription and the expert system to personalize medication. These fourthgeneration systems classified by Gartner "physician colleagues" with advanced analytical capabilities have already begun to spread in the world [49].

Big Data potential in remote monitoring support of patients' health. Big data processing technologies may contribute to the development of personalized and preventive medicine based on remote monitoring of patients. This, in turn, will contribute to the improvement of life quality of the patients through the access to the latest medical services anytime and anywhere, early detection and prevention of unexpected complications in the patient's health, and through the support (automatic reminder) of the patient self-treatment and preventive procedures.

The integration of patients' EHR (medical tests, laboratory tests, prescribed medications, 
information about side effects, contraindications, unstructured text, etc.) with wearable smartdevices with Internet access, controlling the vital health parameters (sleep cycles, heart rate, pressure, etc.), allows physicians to observe the patient in real time. Consolidation of the medical information, which is continuously generated in the process of patient's health monitoring, with EHR data, as well as the simultaneous analysis of the huge volume of diverse data in real time, provide the comprehensive coherent picture of the general health of the patient to the professional. This, in turn, will contribute to informed decision support by remote diagnosis of the patient in real time. At the same time, remote monitoring of patients' health will reduce the costs, as there is no need for laboratory tests, hospitalization, and timely correction of treatment.

Future real time applications of Big Data, such as early detection of infection and the use of preventive measures, can reduce the morbidity and mortality of patients, and even prevent epidemics [10].

According to projections $[36,50]$, the possibility of a real-time analytics of the large-scaled data changing in all areas of medicine can make a revolution in this field.

Big Data features in the promotion of evidence-based medicine. Doctors traditionally use their judgment when choosing the treatment tactics, but a step towards evidence-based medicine has been made in the past few years. Evidence-based medicine is a conceptual approach to the medical practice, involving decisions about the tactics of treatment of the patient basing on reliable results obtained in the course of multiple clinical studies [51]. In other words, evidence-based medicine implies making the best decisions about treatment strategy based on a systematic review of clinical trial data.

Big data has a huge potential for the creation of an evidence base to support medical decisions. Thus, evidence-based medicine is based on the findings obtained from randomized controlled trials of a new treatment performed on a limited number of patients. But in reality, there may be quite rare nuances, adversely affecting the final results, which cannot be detected in the course of research on few samples. Combining multiple individual data sets in Big Data algorithms can provide the most reliable evidence in the choice of treatment tactics $[52,53]$.

Big Data potential to facilitate access to new knowledge. Digitization of medical literature also significantly extends the capabilities of doctors' access to the achievements of new research and treatment technologies introduced into the clinical practice. However, the rapid growth in the number of medical discoveries and their prompt appearance in various sources around the world overweight the physical ability of clinicians to familiarize with all the achievements, even in the context of individual diseases. Thus, for example, around 170 thousand clinical trials of drugs are annually held to fight with cancer in the world, however the access to their results is quite limited [40, 54]. Big data may facilitate the access to the latest developments in the international clinical practice, and thus, may empower the physician to quickly acquire new knowledge. Automation of examination and processing of the medical knowledge market will bring information about medical innovations, new drugs invented by leading scientists at any point of the world, to each interested person [55]. In practice of physician, Big Data may allow him to replenish or renew his knowledge by studying the data in realtime, which is collected from a variety of professionals involved in the treatment of patients with similar diseases. Improvement of the search for knowledge through access to high-performance and highly accurate databases containing the patients' health records will allow to offer prophylactic treatment, to identify successful treatment patterns and to reduce the number of medication errors [56].

Big Data opportunities to support patients' decisions on their own health control. As noted above, the digitization of the healthcare industry contributed to the transformation of most of its aspects. The most notable changes have taken place in relations between patients and health care providers: physicians, medical centers, laboratories, etc. The emergence of numerous and multi-functional wearable devices and services has had a significant impact on the imagination of patients on the principles of providing medical services. According to the results of research conducted by consulting company PricewaterhouseCoopers [57], the patients' priorities have changed a bit, and now the priority, on the one hand, is given to uncomplicated access to health services and treatment results, and on the other hand, to 
the need to become an active participant of the treatment process. The concept of "responsible" patient, seeking to know as much as he can about own health and having the opportunity to make his own decisions through the access to medical knowledge obtained from alternative sources, is becoming increasingly popular in the world. This, in turn, stimulates the demand for medical content and multiple patient-oriented services. The policy of many countries, aimed at improving the health of the nation and promoting the right (healthy) lifestyle also contributes to the strengthening of the trend [58].

According to forecasts, the future of medicine involves the personal participation or involvement of the patient, which will be possible as a result of the emergence of new services and tools to monitor their condition. In this situation, it is advisable to provide the patients (individuals) with the opportunities to track and analyze the health status, access to the information on the treatment methods regarding their specific symptoms, allowing them to make reasonable decisions on more efficient management of their own health.

Big Data may contribute to reforming the relationship between the patients and health care providers for increasing the patients' engagement in the treatment process. According to the current model, electronic health records of the patients (EHR) are at the disposal of a medical institution (MI). In the future, this personal health data (PHD) is planned to be at disposal of the patients. Big Data will complement the PHD of patients in EHR with their personal data from various websites and social networks. In general, the further development of solutions in the field of e-health is focused on creating a platform for the transfer and exchange of information, interaction and cooperation among MI, specialist and doctors, medical workers and patients [59].

Big Data potential in forming client-oriented healthcare. At present, health service providers are striving to introduce an effective strategy for improving patient (client) retention processes by using internal, external and analytical sources to provide a unified view of the client. The emergence of new models of doctors and patients relationship due to the digitization of healthcare forced many medical organizations to adopt more client-oriented approach. At the same time, most organizations have an idea of the client based only on the internal data sources. This impedes forming a comprehensive view of the client and also requires the involvement of external data.

Big Data allows supplementing personal health data of patients in EHR with the information obtained from the external sources providing ample opportunities to identify risk factors or socalled lifestyle factors. Accordingly, in a modern digital society, each person generates a huge amount of information on the Internet through personal data on various websites, accounts in social networks, using credit cards, etc. This information allows revealing the lifestyle factors of a person (for example, material condition, education level, habits, interests, possible diseases, etc.) without the need for interviewing the latter. Cooperative use of "network" information and the patient data from the EHR provides a unique opportunity to integrate traditional medical models to the social determinants of the patient's health and the formation of client-centered healthcare [60].

Big Data potential in managing the treatment of the patients with chronic diseases. Over the past few decades, there has been a significant increase in the number of the patients with chronic diseases almost all over the world. The indicators of comorbidity, i.e., simultaneous presence of two or more chronic diseases in the patient have also significantly increased [61]. This requires the patients to be monitored not only by their primary physician, but also by other specialists. However, the disparity of both health facilities that provide different services to the same patients and the treatment methods reduces the ability of the health system to provide quality health services and leads to unnecessary duplication of analyzes and treatments. In this situation, the patients requiring comprehensive medical care are at greatest risk.

For example, a patient with congestive heart failure, diabetes and chronic lung disease needs a comprehensive treatment that ensures the coordination and the balance of various therapies. However, in practice, such coordination is rarely carried out. Instead, the patients often receive contradictory recommendations from various doctors.

For today, the patients suffering from chronic diseases are the largest consumers of health 
resources. For example, their share in the US health care accounts for $75 \%$ of the costs of this sphere [62]. Big Data can play a decisive role in reducing the costs of the treatments of the patients with chronic diseases. Consequently, using data mining methods, the most similar cohorts of patients perceived as the candidates for a preventive intervention in a high-risk group (Highriskgroup, HRG) can be found [63]. This may reduce health care costs by: 1) improving the coordination of the patients' care; 2) detecting the data duplication and clarifying the therapy; 3 ) reducing repeated hospitalizations through the identification of lifestyle factors (hereditary, socioeconomic, professional, environmental, behavioral, etc.), which increase the risk of deviations in health status; 4) forming the client-centered healthcare.

\section{Big Data opportunities in organizational and managerial decision support}

Analysis of existing medical practice using the Big Data technologies may reduce the hospital costs, eliminate many misapplication and extra costs in public health systems. Thus, MIs may reduce the costs by using huge volumes of diverse data about the patients and by optimizing the work of clinics: predicting the expected flow of the patients, reducing the queues, optimizing the resource provision, assessing the medical personnel performance and its workload balance, improving the patient satisfaction with high quality of medical services and etc [64].

With the help of Big Data technologies, it is possible to improve and regulate the pricing and payment system. For example, in the United States, in accordance with the new federal laws in MIs covered by the Medicare program, the performance of medical personnel is directly related to the payments and fines for medical services measured by such factors as patients' expenditures, their satisfaction, rehospitalization and mortality. Approximately $90 \%$ of medical services in the US is predicted to be paid under the Medicare program by 2018 [65], i.e. depending on the results of the treatment. The shift to the introduction of the payment and pricing system based on a joint assessment of the performance of medical personnel, the cost of medicines and labor, the real financial capacity of the population and the need for services, is possible only on the basis of the systems working with large-scale data.

MIs are tending to improve the quality of patient care while reducing the costs. To improve their performance, MIs are trying to use and analyze large volumes of heterogeneous internal and external data about the patient: clinical data, EHR data, monitoring data of medical devices, data continuously received from monitoring systems (lifestyle devices), and so forth. This enables them to improve the quality and diversity of health services provided to heterogeneous groups of patients. The growing involvement of patients in their own healthcare and the demand for remote health services enables MIs to agree on better payment terms by payers (patients, insurance companies and regulator) [66]. Big Data technologies can also play an important role in extracting and providing valuable information from a huge amount of data to health care providers and decision makers to develop strategies, plans and make important management decisions.

\section{Advantages of Big Data and proper analytics to support appropriate medical decisions}

Generalized advantages of Big Data in medicine can be expressed in their assistance to improve the quality of medical services and additional cost reductions through:

- the possibility of analyzing medical datasets, and therapeutic and diagnostic decision support both in the context of the entire population and its geographic sections, and across different cohorts and individual patients;

- the development of evidence-based medicine by supporting more informed treatment strategies;

- the development of personalized medicine based on an individual approach to the prevention, diagnosis and treatment of genetic diseases;

- automated consolidation of patient's data from the complex heterogeneous sources and its sharing in real time;

- identifying the most similar patient cohorts, who need complex medical care, and 
coordinated decision support on their treatment;

- making organizational and management decisions to reduce costs, modernize the payment system and pricing.

\section{Challenges in using Big Data-analytics in medicine}

Along with the potential of Big Data and growing trend of practical use of the latter, there are certain challenges to their widespread introduction in medicine. Thus, an effective Big Dataanalytics prevent are prevented by:

- continuous growth of medical information, only a small portion of which is used all over the world;

- dominance of unstructured, incomplete, inaccurate and accidental medical data in EHR system, affecting the precision of the information (inaccurate digital input of medical appointments in recipes due to poor handwriting, poor quality of the information obtained from remote sources, the lack of information in one or more fields of EHR, increased garbage, etc.) $[36,67,68]$;

- complication of the exact identification of the patient's identity when comparing the data obtained from a variety of sources (EHR, non-clinical data from social media, web sites, etc.) in order to form a complete picture of the patient's health $[38,69,70]$;

- growing demand for processing continuously generated data of the patient and the generation of analytics in real time, which requires the implementation of complex integration processes that are not available to many clinics due the high cost of Big Data technologies, and the lack of appropriate specialists in the IT departments of MI. The latter must also ensure the safety, reliability and high availability of data from different sources [71];

- potential threats to privacy and information security of personal medical data, related to the risk of misuse of the latter. Another serious threat to the security of personal health data occurred after the wide availability of genetic information, clearly indicating the certain patient. Since this information is almost impossible to anonymize, and deidentified genome data is easy to be recovered, then the privacy issues need to be resolved at the legislative level $[37,72,73]$;

- insufficient volume of accumulated information resources due to lack of EHR and EMR, various warehouses of medical data in many countries;

- lack of specialists with deep analytical knowledge to work with Big Data (Data Scientists), which is estimated to reach 140-190 thousand people by 2018 [4, 74];

- $\quad$ high cost and complexity of adaptation tools, methods and algorithms for the analysis of Big Data limit the scope of healthcare organizations that have the ability to implement these technologies. Additional obstacle to the implementation of Big Data is a need for the continuous financial support for databases update. For example, IBM invested about 3 billion USD in the development of Watson, and supercomputer took "training course" only on a few diseases [75].

\section{Technologies and methods of Big Data processing and analyzing}

In accordance with [76, 77], the term "Big Data technology" implies the approaches, sets of tools (platforms), methods and techniques (procedures) for the processing of huge volumes of multiple data in various formats to achieve the results to be perceived by human.

Methods and techniques of Big Data analysis. Quite a wide spectrum of methods and techniques have been developed and adapted for the unification, storage, manipulation, analysis and visualization of Big Data. These methods and techniques are borrowed from many fields, including statistics, computer science, artificial intelligence, applied mathematics, linguistics, etc. Without pretending to be exhaustive, we list some of the methods and analysis techniques of Big Data, allocated in the works [4, 43, 78]: Data Mining methods, crowdsourcing, integration of heterogeneous data, visualization, statistical analysis, predictive analytics, predictive modeling, 
machine learning, natural language processing, artificial neural networks, learning association rules, classification, cluster analysis, regression analysis; artificial neural networks, network analysis, optimization, genetic algorithms; pattern recognition, etc.

Big Data processing and analysis tools. The basic requirements to the development of software systems for working with big data is the inclusion of parallel processing tools and distributed data storage, as well as the transition from models used in traditional relational databases to the new models supporting the constant change of data structures, and the horizontal scaling through the clusters of inexpensive commodity computers, etc. For example, a new class of NoSQL systems (Not Only SQL), such as HBase, Cassandra, MongoDB, Neo4j, Riak and etc. are considered the development of advanced relational SQL databases [79].

The most common Big Data processing systems are Hadoop platform and distributed computing paradigm MapReduce. Hadoop is capable to handle very large volumes of heterogeneous input data mainly through the distribution of the latter on multiple servers (nodes), each of which produces the processing of a specific set of data, and then the results of the subtasks are reduced to the final (conclusive) result. This enables the use of Hadoop for the implementation of search and contextual mechanisms of heavily loaded sites, as Yahoo, Facebook, Amazon, and etc. Making Hadoop code public by Yahoo has contributed to the emergence of the whole field of production, based on Hadoop, in IT industry, the developers of which are both well-known global companies and start-ups.

In recent years, many software products have been developed that facilitate the work with Big Data, including SAP HANA, high-performance platform NewSQL for data storage and processing, the applications for pre-processing of information flows (Marshalling), solutions for visualization and self-analysis (Advanced Analytics with Self -Service Delivery), and others. The most popular programming languages are Python, Java and Scala.

Availability of Big Data technologies has significantly increased due to cloud computing, which provides a new level of processing speed [80]. There are many cloud services in Big Data analytics, and cloud platforms for application deployment. With their help, Big Data analytics can be uses "anytime and anywhere", simply by connecting to the Internet and selecting the appropriate service.

It should be noted that, today, in the process of introduction of the newly developed software in medicine, the users face a number of significant shortcomings associated with the specifics of the industry, which need to be addressed. Furthermore, the offered platforms/tools require specialized programming skills, which is not typical for an end user of health care. Since Big Data analysis technology has been recently used in medicine, a number of problems related to the data management, privacy, security, standardization, etc. have to be resolved.

\section{Development prospects of Big Data in medicine}

According ABI Research, currently, the processes of Big Data analysis in medicine are in the early stages. However, according to the forecasts, as a result of the rapid growth of continuously generated data, the market of Big Data-analysis will make up approximately 52 billion USD by 2019 [71]. TechNavio analysts predict the increase in the cost of the global Big Data market in the field of medicine for 2014-2019, with an average annual growth rate of 42\% [81].

Here are the most promising areas of development and implementation of Big Data in medicine [82-86]:

- development of mobile medicine, which expands the capabilities of remote diagnosis and continuous monitoring of the health status of certain categories of patients, that actualizes the demand for streaming processing of the information through the development of analytics basing on the patient's data set in real-time;

- promoting technologies to support informed diagnostic and treatment decisions based on a comparative analysis of the patient's data with the EHM databases containing the treatment practices of similar diseases and identifying the most suitable treatment method in each case;

- expanding the introduction of Big Data in the realization of personalized medicine; 
- expanding the basic research in the field of optimal methods of processing, analyzing and extracting useful knowledge from large volumes of medical data of various nature;

- personnel training to handle big medical data;

- development and adaptation of the tools to handle Big Data for the medical industry: development trends in the field of mobile applications, cloud infrastructure, data visualization, etc.

- Big Data-solutions for the processing of medical data generated by "smart" devices within the framework of the exponentially growing Internet of Things.

\section{Conclusion}

Conducted analysis of the status of Big Data in medicine shows that, at present, this segment of the market is in its early development stage. However, all the growing digitalization of medicine, the conversion of the Internet into one of the most powerful medical information sources and infrastructure for fast, reliable and secure data transmission, the development of social networks, mobile and wireless access to health information have contributed to the transformation of many aspects of the medical industry, and have led to the emergence of new trends. We can point out a number of new trends, such as: 1) development person-centered medicine, implying effectively organized access to any set of medical records and the primary results of the patient's examination; 2) changing role of patients, their increasing involvement in their own health care and the possibility of self-monitoring; 3) changing methods of interaction between doctors and patients, and the access of the latter to health services, treatment results and medical information; 4) delivery of medical services in real time; 5) development of personalized medicine, based on the individual patient sensitivity to drugs, when appointing the therapy, etc. All these transformations are taking place at the background of a common goal, focused on improving the efficiency and flexibility of the health care system by increasing the quality of medical services and reducing costs.

Big Data can have a significant impact on the healthcare. The enormous amount of data currently generated by medical institutions will grow at an even greater rate in the coming years. This will inevitably lead to an increase in demand for Big Data analysis. Extraction of the useful information from this data can be of great importance for the development of new methods and treatment technologies, the identification of diseases and their prevention, reducing the number of medical errors, ensuring the total security of the health of the population, perfecting the health care system towards the evidence-based and personalized medicine. This will result ultimately in improved treatment and reduced number of patients and consequently, medical facilities costs.

It should be noted that, although potentially Big Data in medicine can help to address many problems, today, there are very few actual examples of successful application of this new technology in medicine. Analysts and practitioners see the reason in the lack of adaptation of Big Data platforms available on the market to the specific characteristics of the healthcare industry, to the requirements of confidentiality and security of medical data, as well as to the standardization of medical solutions, etc. Implementation of Big Data in medicine is also affected by the factors, such as distrust of doctors and the uncertainty of a number of IT companies, in the effectiveness of these technologies. This means that the use of Big Data in medicine passes through the solution of mentioned problems and the improvement of these technologies. The development dynamics of Big Data technologies and services, as well as the analysts' forecasts reinforce the confidence that, in the near future, there will be a significant increase in the application of Big Data-solutions in medicine. Moreover, according to latter ones, the further digitalization will inevitably pave the way to revolutionary changes in medicine, the consequences of which will be much bigger. 


\section{References}

1. https://ru.wikipedia.org/wiki

2. The Australian public service big data strategy. Canberra: Commonwealth of Australia 2013 www.aiia.com.au/documents/policy-submissions/policies-and-submissions/2014/pdf

3. The growth of information volume - realities of the digital universe. ww.tssonline.ru/articles2/fix-corp/rost-obema-informatsii-realii-tsifrovoy-vselennoy

4. James Manyika, Michael Chui, Brad Brown, Jacques Bughin, Richard Dobbs, Charles Roxburgh, Angela Hung Bayers. Big data: The next frontier for innovation, competition, and productivity. Analyst report, McKinsey Global Institute, May 2011. www.mckinsey.com

5. Raghupathi W. Data Mining in Health Care /Healthcare Informatics: Improving Efficiency and Productivity. Edited by Kudyba S. Taylor \& Francis; 2010:211-223.

6. Sun, J., Reddy, C. Big data analytics for healthcare. International Conference on Data Mining. Austin, TX., 2013. www.siam.org/meetings/sdm13/sun.pdf

7. Manchini M. Exploiting Big Data for improving healthcare servuces// Journal of e-Learning and Knowledge Society, 2014, v.10, n.2, pp.23-33.

8. Roski J, Bo-Linn GW, Andrews TA. Creating value in health care through big data: opportunities and policy implications. Health Affairs, 2014 Jul.33(7):1115-1122. www.ncbi.nlm.nih.gov/pubmed/25006136

9. Datta M. How Big Data Will Lower Costs and Advance Personalized Medicine. GEN Exclusives, 2013. www.genengnews.com/insight-and-intelligence/how-big-data-willlower-costs-and-advance-personalized-medicine/77899962/

10. Transforming Health Care through Big Data. Strategies for leveraging big data in the health care industry. Institute for Health Technology Transformation, 2013. http://c4fd63cb482ce6861463-bc6183f1c18e748a49b87a25911a05 /iHT2BigData2013.pdf

11. Iain Buchan, Chris Bishop. A Unified Modelling Approach to Data-Intensive Healtcare. The fourth paradigm. 2009, pp.91-97.

12. Powell J., Buchan I. Electronic health records should support clinical research // Journal of Medical Internet Research, 2005, vol.7, no.1, p.e4, doi: 10.2196/jmir.7.1.e4.

13. Zingerman B. Electronic medical records and its organization principles. www.osp.ru/medit/blogs/bz/bz_109.html

14. Kashmir H. How Target Figured Out A Teen Girl Was Pregnant Before Her Father Did // Forbes, 2012. http://ghr.nlm.nih.gov/glossary=personalizedmedicine

15. Personalized medicine. http://ghr.nlm.nih.gov/glossary=personalizedmedicine

16. Jain K.K. Personalized medicine// Advances in Clinical Chemistry, 2008; №4, pp.548-558.

17. Gillam M., Feied C, Handler J., Moody E., Shneiderman B., Plaisant C., Smith M., Dickason J. The Healthcare Singularity and the Age of Semantic Medicine. The fourth paradigm. 2009, pp.57-64

18. Davenport T., Glaser J., Just-in-time delivery comes to knowledge management, Harvard Business Review, 2002, vol.80, no.7, pp.107-111, 126doi: 10.1225/R0207H.

19. Manhattan research. Social Media\&Health 2.0. http://manhattanresearch.com/ResearchTopics/Consumer/HealthSocial-Media.

20. Dave Evans. Internet of things: how our whole life will change in the next development stage of the network. www.cisco.com/web/RU/news/releases/txt/2011/062711d.html

21. Internet-of-Everything makes everything possible... http://www.internet-of-everything.no/

22. Gawande A. Annals of Health Care. NewYorker, 2012. www.newyorker.com/reporting/2012/08/13/120813fa_fact_gawande

23. mHealth: New horizons for health through mobile technologies.WHO. Global Observatory for eHealth series, Vol. 3, 112p. http://www.who.int/goe/publications/goe_mhealth_web.pdf.

24. The world in 2010: ICT facts and figures. Geneva, International Telecommunications Union, 
2010 (http:// www.itu.int/ITU-D/ict/material/FactsFigures2010.pdf)

25. White T. Hadoop: The Definitive Guide. OReilly Media, $3^{\text {rd }}$ Edition, 2012.

http://cdn.oreilly.com/oreilly/booksamplers/9781449311520_sampler.pdf

26. http://en.wikipedia.org/wiki/Big_data

27. Knowledge management in organizations // Proceedings of 9th International Conference, KMO 2014, Santiago, Chile, September 2-5, 2014. http://www.springer.com/gp/book

28. What Is The Difference Between Telemedicine, Telecare and Telehealth? http://evisit.com/what-is-the-difference-between-telemedicine-telecare-and-telehealth/

29. Telehealth, Telecare and Telemedicine...What's the Difference?

www.globalmed.com/additional-resources/telehealth-telecare-and-telemedicine.php

30. https://ru.wikipedia.org/wiki

31. Lagutin Y. Physicians interested in expert knowledge bases. www.computerra.ru/cio/497

32. Hesla L. Particle physics tames big data// Symmetry magazine, 2012. www.symmetrymagazine.org/article/august-2012/particle-physics

33. Laney D. 3D Data Management: Controlling Data Volume, Velocity and Variety // Application Delivery Strategies. META Group. 2001. http://blogs.gartner.com/douglaney/files/2012/01/ad949-3D-Data-Management-Data-Volume-Velocity-and-Variety.pdf

34. Savelyev A.I., Problems of introducing the legislation on personal data in the era of "big data" (BIG DATA). http://www.hse.ru/pubs/share/direct/document/150345956).

35. Herman R, Williams P. Big Data in healthcare: what is it used for? http://ro.ecu.edu.au/cgi/viewcontent.cgi?article $=1021 \&$ context=aeis

36. Feldman B., Martin E., Skotnes, T. Big data in healthcare: Hype and hope. 2012. www.west-info.eu/files/big-data-in-healthcare.pdf

37. Mattison J. How to Navigate Big Data in Healthcare. www.cio.com/article/2851986/healthcare/how-to-navigate-big-data-in-healthcare.html

38. Panahiazar M., Taslimitehrani V., Jadhav A., Pathak J. Empowering personalized medicine with Big Data and Semantic Web Technology // Proc IEEE Int Conf Big Data. 2014: 790-795. www.ncbi.nlm.nih.gov/pmc/articles/PMC4333680/.

39. LaValle S., Lesser E, Shockley R., Hopkins MS., Kruschwitz N.:Big data, analytics and the path from insights to value. MIT Sloan Management Review, 2011, vol.52, no.2. www.ibm.com/smarterplanet/global/files/in_idea_smarter_computing_to_big_data.pdf

40. Raghupathi W., Raghupathi V. Big Data Analytics in Healthcare: Promise and Potential// Health Inform. Science and Systems, 2014, 2:3. www.hissjournal.com/content/2/1/3

41. How Data Analytics can help in Decision Making in Healthcare. A Saviance Technologies Whitepaper. http://sharinghealthcaresolutions.covidien.com/how-do-you-use-big-data-drive

42. IBM's Watson Gets Its First Piece Of Business In Healthcare www.forbes.com/sites/bruceupbin/2013/02/08/ibms-watson-gets-its-first-piece-of-bus

43. Krylov V.V., Krylov S.V. Big data and their applications in the power industry. Pub. Nobel Press, 2014, 166 p.

44. Hoffman S. Medical big data and big data quality problems. http://insurancejournal.org/wpcontent/uploads/2015/03/Hoffman.pdf

45. Hood L. Systems biology and p4 medicine: past, present, and future // Rambam Maimonides Med. J. 2013, vol. 4 (2). p. e0012. doi: 10.5041/RMMJ.10112

46. Sacchi L., Lanzola G., Viani N., Quaglini S., Personalization and Patient Involvement in Decision Support Systems: Current Trends // IMIA Yearbook of Medical Informatics, 2015, vol. 10 (1), pp.106-118

47. Horvitz E. From Data to Predictions and Decisions: Enabling Evidence-Based Healthcare. Computing Community Consortium. 2010. http://www.cra.org/ccc/initiatives

48. PMC Analysis. 2015. http://www.personalizedmedicinecoalition.org/News/Press_Releases/

49. Generations of the IIA according to Gartner, 2013. https://ru- 
ru.facebook.com/OLTechnologies/posts/8162

50. Basel Kayyali, David Knott, and Steve Van Kuiken. The Big-Data revolution in Healthcare: Accelerating value and innovation. Full report. 2013.

www.mckinsey.com/insights/health_systems_and_services/big-data_in health_care

51. Evidence Based Medicine: A new paradigm for the patient. Evidence Based Medicine Working Group, 1993 http://jama.jamanetwork.com/article. aspx?articleid=404143/.

52. Murdoch T.B.; Detsky A.S. The Inevitable Application of Big Data to Health Care// Journal of the American Medical Association. 2013; 309(13):1351-1352. http://jama.jamanetwork.com/article.aspx?articleid=1674245

53. Discovery with Data: Leveraging Statistics with Computer Science to Transform Science and Society. www.amstat.org/policy/pdfs/BigDataStatisticsJune2014.pdf

54. Alper B.S., Hand J.A., Elliott S.G., Kinkade S., Hauan M.J., Onion D.K., Sklar B. M. How much effort is needed to keep up with the literature relevant for primary care? //Journal of the Medical Library Association, 2004, vol.92, no.4, pp.429-437.

55. Rice S. Using big data to prevent drug errors. 2015 http://www.modernhealthcare.com/article/20150711/MAGAZINE/307119976

56. Lenfant C. Clinical Research to Clinical Practice - Lost in Translation? The New England Journal of Medicine, 2003, vol.349, pp.868-874, PMID: 12944573

57. Growth through innovation. Russian and international experience www.pwc.ru/innovationsurvey

58. Big data in the healthcare industry: Growing Need for Computerized Decision Support. www.healthcare.siemens.com/magazine/mso-big-data-and-healthcare-1.html

59. Data-driven healthcare organizations use big data analytics for big gains. IBM Software White Paper. 2013. www03.ibm.com/industries/ca/en/healthcare/documents/Data.pdf

60. Davenport T.H., Barth P.,Bean R. How 'Big Data' Is Different / MIT Sloan Management Review/ http://sloanreview.mit.edu/article/how-big-data-is-different/

61. Uijen A.A., Vande Lisdonk E.H. Multimorbidity in primary care: prevalence and trend over the last 20 years // European Journal of General Practice, 2008, vol.14, no.1, pp.28-32.

62. Treatment of patients with multiple chronic diseases. Journal of McKinsey. http://vestnikmckinsey.ru/healthcare-and-pharmaceuticals/Lechenie-patsyentov-smnozhestvom-khronicheskykh-zabolevaniy

63. Bates D.W., Saria S., Ohno-Machado L., Shah A., Escobar G. Big Data In Health Care: Using Analytics To Identify And Manage High-Risk And High-Cost Patients. http://content.healthaffairs.org/content/33/7/1123.full?ijkey=QhqcLkIaGb84M

64. Big Data and Analytics. https://www.mill-all.com/wp-content/uploads/2015/08/Healthcare

65. www.medicare.gov/

66. Mani N., Narayanan R., Raghunath M. Big Data in the healthcare provider space. www.mu-sigma.com/analytics/thought_leadership/decision-sciences.html

67. Challenges and opportunites with Big Data. http://cra.org/ccc/wpcontent/uploads/sites/2/2015/05/bigdatawhitepaper.pdf

68. Hersh W. The Health Information Technology Workforce // Journal Applied Clinical Informatics (ACI), 2010, vol.1, no.2, pp.197-212.

69. A Policy Forum on the Use of Big Data in Health Care, 2014. http://bipartisanpolicy.org/library/policy-forum-use-big-data-health-care

70. Robertson J., Dehart D., Tolle K., Hecherman D. Healthcare Delivery in Developing Countries: Challenges and Potential Solutions The fourth paradigm. 2009, pp.65-73

71. ABI Research: Integrating Consumer Wearable Health Devices Will Drive Healthcare Big Data Adoption. www.abiresearch.com/press/integrating-consumer-wearable-health

72. Mammadova M.G. Information security of personal health data in an electronic environment // Problems of Information Technologies, 2015, No2, pp.16-30. 
http://jpit.az/index.php?mod=9\&view=art\&id=142)

73. Big data security and privacy issues in healthcare www.computer.org/csdl/proceedings/bigdatacongress/2014/5057/00/5057a762.pdf

74. Mammadova M., Jabrayilova Z. Application, opportunities, and challenges of Big data in human resource management // Problems of İnformation Technology 2016, No1, pp.39-48.

75. Mattison on Big Data: Great potential but risks exist. http://www.clinicalinnovation.com/topics/analytics-quality/mattison-big-data-great-potential-risks-exist)

76. Borkar VR, Carey MJ, Chen L: Big data platforms: what's next? // ACM Crossroads 2012, 19(1), pp.44-49.

77. Zikopoulos PC, DeRoos D, Parasuraman K, Deutsch T, Corrigan D, Giles J: Harness the Power of Big Data. The IBM Big Data Platform; McGraw-Hill: 2013, 281p.

78. Aliguliyev R.M., Imamverdiyev Y.N., Abdullayeva F.J. Studying the possibility of implementing big data analytics cloud computing platform analytics-as-a-service in oil and gas industry // Problems of Information Technologies, 2016, No1, pp.11-26.

79. Ian Gordon, John Klein. Systems for Big Data: Architecture Convergence // "Open Systems", 2015, No03. www.osp.ru/os/2015/03/13046898/

80. Big Data in the Cloud: Converging Technologies: How to Create Competitive Advantage Using Cloud-Based Big Data Analytics./Intel IT Center. www.intel.com/content/dam/www/public/us/en/documents/product-briefs/big-data.pdf

81. Global Big Data spending in healthcare 2015 analysis and forecasts to 2019. www.prnewswire.com/news-releases/global-big-data-spending-in-healthcare-industry

82. Big Data\&Analytics. IDC Future Scape: Worldwide Big Data and Analytics 2016 Predictions - APEJ Implications. Dec 2015 https://www.idc.com/prodserv/4Pillars/bigdata

83. The future of healthcare in Europe. The Economist Intelligence Unit Limited 2011, 54 p.

84. The Future of Healthcare Innovation, Big Data, Security and Patient Engagement. http://electronichealthreporter.com/the-future-of-healthcare-innovation-big-data-security

85. How to transform big data into better health. Workshop report, Italy, 2014. www.scienceeurope.org/uploads/publicdocumentsandspeeches/scspublicdocs/report

86. Continuous healthcare: Big data and the future of medicine.

http://venturebeat.com/2015/06/21/continuous-healthcare-bigdata-and-future-of-medicine 